\title{
Compatibility of ingredients of Danshen (Radix Salviae Miltiorrhizae) and Honghua (Flos Carthami) and their protective effects on cerebral ischemia-reperfusion injury in rats
}

\author{
HAOYU WAN ${ }^{1 *}$, YUTING YANG ${ }^{1 *}$, ZHIWEI LI $^{1}$, LAN CHENG $^{1}$, ZHISHAN DING $^{1}$, \\ HAITONG WAN $^{1}$, JIEHONG YANG ${ }^{2}$ and HUIFEN ZHOU ${ }^{1}$ \\ ${ }^{1}$ College of Life Science and ${ }^{2}$ College of Basic Medical Sciences, \\ Zhejiang Chinese Medical University, Hangzhou, Zhejiang 310053, P.R. China
}

Received October 29, 2020; Accepted April 23, 2021

DOI: $10.3892 / \mathrm{etm} .2021 .10281$

\begin{abstract}
Danshen (Radix Salviae Miltiorrhizae) and Honghua (Flos Carthami) (Danhong) are two drugs commonly prescribed together, which are often used in the treatment of cerebrovascular diseases in China. Due to the complexity of the ingredients of Danhong, the present study focused on performing the orthogonal compatibility method on the primary effective molecules of this drug: Tanshinol, salvianolic acid A, salvianolic acid B and hydroxysafflor yellow A. These four molecules were studied to determine their protective effects and to screen for the most compatible ingredients to improve cerebral ischemia-reperfusion injury (IR) in rats. Focal middle cerebral artery occlusion was performed to establish the cerebral IR model in rats. Male Sprague-Dawley rats were randomly divided into sham operation group, IR group and nine orthogonal administration groups with different ratios of Danhong effective ingredients and Danhong injection group. Neurological deficit score and cerebral infarction volume were measured postoperatively. Morphological pathological alterations were observed via $\mathrm{H} \& \mathrm{E}$ staining. Bcl-2 and Bax were quantified using ELISA. Immunohistochemistry was conducted to analyze the expression of caspase- 3 in the hippocampus. The expression levels of cytochrome $c$, apoptotic peptidase activating factor 1 (apaf-1),
\end{abstract}

Correspondence to: Dr Huifen Zhou, College of Life Science, Zhejiang Chinese Medical University, 548 Binwen Road, Hangzhou, Zhejiang 310053, P.R. China

E-mail: zhouhuifen2320@126.com

Professor Jiehong Yang, College of Basic Medical Sciences, Zhejiang Chinese Medical University, 548 Binwen Road, Hangzhou, Zhejiang 310053, P.R. China

E-mail:yjhong@zjtcm.net

*Contributed equally

Key words: Danshen, Honghua, effective ingredients, cerebral ischemia-reperfusion injury, apoptosis caspase-9, caspase-3 and p53 mRNA in the hippocampus were assessed via reverse transcription-quantitative PCR. The results demonstrated that different compatibility groups significantly reduced the neurological function score and decreased the volume of cerebral infarct compared with the IR group. These groups were also indicated to improve the pathological damage to the brain tissue. In addition, certain compatibility groups significantly decreased the number of caspase-3 positive cells in the hippocampus and the expression levels of cytochrome $c$, apaf-1, caspase-9, caspase-3 and p53 mRNA in the brain tissue. Orthogonal group $4(30 \mathrm{mg} / \mathrm{kg}$ tanshinol; $2.5 \mathrm{mg} / \mathrm{kg}$ salvianolic acid A; $16 \mathrm{mg} / \mathrm{kg}$ salvianolic acid B; $8 \mathrm{mg} / \mathrm{kg}$ hydroxysafflor yellow A) was indicated to be the most effective. The four effective ingredients of Danhong exhibited a protective effect on rats with cerebral IR injury, potentially through the inhibition of apoptosis via the downregulation of key targets upstream of the caspase- 3 pathway. In addition, the present study provided novel insights for the continued study of the drug compatibility rules of TCM.

\section{Introduction}

Stroke has become the main clinical type of cerebrovascular disease, which is a type of disorder of blood circulation in brain tissues (1). Pathologically, stroke can be divided into ischemic stroke and hemorrhagic stroke (2). More than $80 \%$ of the global burden of stroke is attributed to ischemic stroke (3). Ischemic strokes often present with high rates of incidence, recurrence, disability and mortality for patients (4). In 2008, an epidemiological survey indicated that strokes, with an incidence of 136.64 per 100,000 individuals, had replaced cancer as the leading cause of mortality in China (5). At present, intravenous thrombolytic therapy is the main clinical treatment for ischemic stroke $(1,6)$, and there is still a lack of effective drugs to protect neurons from death. Therefore, there is a need for multi-target and improved therapeutic drugs, which is why the beneficial effects of Traditional Chinese Medicine (TCM) is worth investigating (7).

Danshen and Honghua (Danhong) are classic blood-activating drugs often used for promoting blood circulation and believed to remove blood stasis in TCM. They have a long 
history in the treatment of cardiovascular and cerebrovascular diseases in traditional clinical trials (8-11). With the progress of modern research and separation technology, it has been revealed that the primary effective ingredients in Danshen are tanshinol, salvianolic acid $\mathrm{A}$ and salvianolic acid B. These water-soluble molecules were indicated to exhibit a variety of favorable effects, including neuroprotective activity, antioxidation, regenerative effects and responses similar to those of an antidepressant (12-14). Hydroxysafflor yellow A is the main bioactive component in Honghua, which could protect against ischemic stroke by promoting the dilation of cerebral vessels to improve cerebrovascular permeability (15-17). In addition, these four molecules displayed protective and regulatory effects on disturbed metabolism and the regulation of neuroinflammatory responses (17-22).

Collectively, the four effective ingredients of Danhong were indicated to attenuate cerebral ischemic injury in vitro (23). In the present study, the orthogonal compatibility of the four effective ingredients of Danhong (tanshinol, salvianolic acid A, salvianolic acid B and hydroxysafflor yellow A) were examined to explore the protective effect of Danhong on cerebral ischemia-reperfusion (IR) injury in rats. The current study aimed to provide novel insights and guidance for the clinical and experimental treatment of ischemic cerebrovascular disease.

\section{Materials and methods}

Animals. Healthy adult male Sprague-Dawley rats (total, 216; weighing 260-300 g) with clean grade were purchased from Zhejiang Laboratory Animal Center. Animal license number was SCXK (Zhejiang) 2014-0001. The temperature of the animal room was controlled at $25 \pm 1^{\circ} \mathrm{C}$, and air humidity was 60-65\%. The rats were placed in a 12:12 h light/dark cycle with access to food and water ad libitum. The rats were euthanized via cervical dislocation under pentobarbital sodium anesthesia [1\% in normal saline (NS); $35 \mathrm{mg} / \mathrm{kg}$; intraperitoneally administered].

Chemicals and reagents. Danhong injection was supplied by Shandong Buchang Pharmaceuticals Co., Ltd. 2,3,5-triphenyltetrazolium chloride (TTC) and H\&E were obtained from ShangHai SSS Reagent Co., Ltd. Xylene was purchased from Huadong Medicine Co., Ltd. Rat Bcl-2 (cat. no. MB-7297B) and Bax ELISA kits (cat. no. MB-6629A) were obtained from Shanghai YuanYe Biotechnology Co., Ltd. DAB chromogenic kit (cat. no. ZLI-9018) was obtained from Beijing Zhongshan Jinqiao Biotechnology Co., Ltd. TRIzol ${ }^{\circledR}$ reagent (cat. no. 15596-026) and caspase-3 antibody (1:100 dilution; cat. no. 43-7800) were purchased from Thermo Fisher Scientific, Inc. Tanshinol (purity $>98 \%$; cat. no. 76822-21-4; batch. no. SZ201707038), salvianolic acid A (purity >98\%; cat. no. 96574-01-5; batch. no. SZ201706001), salvianolic acid B (purity >98\%; cat. no. 121521-90-2; batch. no. SZ201706003) and hydroxysafflor yellow A (purity $>98 \%$; cat. no. 78281-02-4; batch. no. Z201702005) were obtained from Nanjing Shizhou Biotechnology Co., Ltd.

Instruments. The instruments used in the present study were as follows: OHAUS AR153CN electronic balance (OHAUS
Instruments Shanghai Co., Ltd.), analytical balance (Mettler Toledo), Pall Cascada Bio Mk2 Water Filtration system (Pall Life Sciences), fluorescence quantitative PCR instrument (Bio-Rad Laboratories, Inc.), ZH-003 stainless steel brain matrices (Anhui Zhenghua Biological Equipment Co., Ltd.), Rotary Microtome Microm HM 340E (Thermo Fisher Scientific, Inc.) and Leica DM LB2 microscope camera (Leica Microsystems GmbH).

Transient focal cerebral ischemia model. The experimental procedure was developed and performed after certain adjustments to the method by Longa et al (24). The rats were anesthetized intraperitoneally with $1 \%$ pentobarbital sodium (35 mg/kg). Their body temperature was kept constant at $37^{\circ} \mathrm{C}$. The rats were immobilized in the supine position and sanitized with alcohol before the skin was removed. A median longitudinal incision was made on the neck. The superficial fascia was cut from the bilateral submandibular glands to expose one side of the mastoid muscle. The muscle gap was bluntly separated between the right sternocleidomastoid muscle and the sternohyoid muscle to expose the right side. This allowed for visualization of three major blood vessels: The right common carotid artery (CCA), external carotid artery (ECA) and internal carotid artery (ICA). The root of the ECA and the proximal end of the CCA were ligated and the ICA was clamped with an arterial clip. Subsequently, a nylon wire with a smooth rounded tip (diameter, 0.28-mm; Beijing Cinontech Co., Ltd.) was inserted from CCA into ICA gently, and the arterial clip was removed. The insertion depth was stopped at the origin of the middle cerebral artery $(18-20 \mathrm{~mm})$, and the ischemic time was recorded. After ischemia for $1 \mathrm{~h}$, the wire was gently withdrawn for reperfusion, and rats were euthanized 3 days after. The incision was sutured layer by layer and disinfected. The rats were returned to their cages and kept in the lateral position after the operation, and their body temperature was maintained at $37^{\circ} \mathrm{C}$.

Groups and treatment. Sprague-Dawley rats were randomly divided in one of 12 groups: Sham operation (sham), IR untreated model (IRU), Danhong injection group (DHI) and orthogonal groups $\left[\mathrm{L}_{9}\left(3^{4}\right)\right]$. The nine different combinations of the four key ingredients of Danhong were prepared according to orthogonal experimental design $(25,26)$, which is a design method to study multi-factors and multi-levels. The orthogonal design is presented in Table I. For example, Group 1 is made of four components at dose $1(15 \mathrm{mg} / \mathrm{kg}$ tanshinol, $2.5 \mathrm{mg} / \mathrm{kg}$ salvianolic acid A, $8 \mathrm{mg} / \mathrm{kg}$ salvianolic acid $\mathrm{B}$ and $2 \mathrm{mg} / \mathrm{kg}$ hydroxysafflor yellow A). The doses of the four individual components were all within the safe range according to previous pharmacological research and related literature (16-22).

Each group contained 18 rats (six rats were used for TTC staining, H\&E staining and immunohistochemistry and PCR, respectively). Firstly, the drug was dissolved in physiological saline. Subsequently, the orthogonal group dose was administered to the tail vein of the $\mathrm{L}_{9}\left(3^{4}\right)$ groups directly at $0 \mathrm{~h}$ after reperfusion. Sham and IRU groups were administered an equal amount of physiological saline. The positive control group was administered Danhong injection $(2 \mathrm{ml} / \mathrm{kg})(27-29)$. 
Table I. Doses of nine compatibility groups of four effective ingredients according to $\mathrm{L}_{9}\left(3^{4}\right)$.

\begin{tabular}{lccrr}
\hline & \multicolumn{4}{c}{ Dose $(\mathrm{mg} / \mathrm{kg})$} \\
\cline { 2 - 5 } Group & $\mathrm{A}$ & $\mathrm{B}$ & $\mathrm{C}$ & $\mathrm{D}$ \\
\hline 1 & 15 & 2.5 & 8 & 2 \\
2 & 15 & 5 & 16 & 4 \\
3 & 15 & 10 & 24 & 8 \\
4 & 30 & 2.5 & 16 & 8 \\
5 & 30 & 5 & 24 & 2 \\
6 & 30 & 10 & 8 & 4 \\
7 & 60 & 2.5 & 24 & 4 \\
8 & 60 & 5 & 8 & 8 \\
9 & 60 & 10 & 16 & 2
\end{tabular}

A, tanshinol; B, salvianolic acid A; C, salvianolic acid B; D, hydroxysafflor yellow A.

Neurological assessments. Assessments of neurological function were performed following reperfusion in accordance with previously described methods (24). Neurological function was assessed using the modified five-point scale scoring system ranging from 0 to 4 , with higher scores being indicative of a more severe neurological impairment. Rats with scores of 1-4 following MCAO were used for analysis.

Measurement of infarct volume. Rats were euthanized under anesthesia on the 3rd day after surgery for TTC staining. The rat brains were cut into small sections $(2.0 \mathrm{~mm})$, immersed in $2 \% \mathrm{TTC}$ at $37^{\circ} \mathrm{C}$ for $30 \mathrm{~min}$. Areas of red staining indicated normal brain tissue, and pale gray areas represented infarcted tissue. Image-Pro Plus v6.0 software (Media Cybernetics, Inc.) was used to calculate the infarct volumes. The following formula was used to calculate cerebral infarction rate: Infarct Rate $=$ Infarct Volume/Whole Brain Volume $\mathrm{x} 100 \%$.

$H \& E$ staining. A total of 3 days after cerebral IR, the rats were anesthetized with $35 \mathrm{mg} / \mathrm{kg}$ pentobarbital sodium and then fixed with $200 \mathrm{ml}$ 4\% paraformaldehyde via perfusion of the heart until the right atrial appendage produced clear liquid. The rats were decapitated, and the brains were fixed in $4 \%$ paraformaldehyde $(\mathrm{Ph} 7.4)$ for $24 \mathrm{~h}$ at $4^{\circ} \mathrm{C}$. After gradient elution (100 and 95\% ethanol for $5 \mathrm{~min}$, respectively), brain tissues were embedded in paraffin and serially sliced (3-4 $\mu \mathrm{m})$. Subsequently, the slices were immersed in hematoxylin for $5 \mathrm{~min}$ and eosin for $2 \mathrm{~min}$ at room temperature. The results of H\&E staining were observed under a light microscope (magnification, $\mathrm{x} 100$ ).

Measurement of Bcl-2 and Bax levels in serum. At day 3 after MCAO, the rats were deeply anesthetized with $35 \mathrm{mg} / \mathrm{kg}$ pentobarbital sodium. A total of $\sim 6 \mathrm{ml}$ blood was drawn from the abdominal aorta and subsequently centrifuged at $1500 \mathrm{x} \mathrm{g}$ for $15 \mathrm{~min}$ at $4^{\circ} \mathrm{C}$. The levels of Bcl-2 and Bax in the serum were measured via ELISA using commercially available kits according to the manufacturer's instructions.
Immunohistochemistry. After fixation, embedding and routine paraffin sectioning of 3-4- $\mu \mathrm{m}$ as aforementioned, the experiment followed the procedure of DAKO En Vision ${ }^{\mathrm{TM}}$ two-step immunohistochemistry kit (cat. no. K5007; Hangzhou Xincheng Biotech Co., Ltd.) (30). Under a light microscope (magnification, $\mathrm{x} 200$ ), the positive cell status of immunohistochemistry was shown as yellow or yellow brown in the cytoplasm. The staining result was determined based on immunoreactivity score (31) by multiplying the intensity of staining $(0=$ not stained; $1=$ low intensity; $2=$ moderate intensity; $3=$ high intensity) and the percentage of immune positive cells $(0=$ not stained; $1=1-10 \% ; 2=11-50 \% ; 3=51-80 \% ; 4 \geq 80 \%)$.

Reverse transcription-quantitative PCR (RT-qPCR) analysis. Frozen brain tissue was placed in a centrifuge tube and the RNA from the right hippocampus of each group of rats was extracted with TRIzol ${ }^{\circledR}$ reagent. RNA concentration and purity were determined using a NanoDrop 2000 spectrometer (Thermo Fisher Scientific, Inc.). The extracted RNA was then reverse transcribed into cDNA using a ThermoScript RT-PCR system (cat. no. 11146016; Toyobo Life Science) according to the manufacturer's instructions. The target genes and GAPDH internal reference gene (Sangon Biotech Co., Ltd.) were amplified by an Applied Biosystems 7500 Fast RT-PCR system (Applied Biosystems; Thermo Fisher Scientific, Inc.). The reaction conditions were as follows: $94^{\circ} \mathrm{C}$ for $3 \mathrm{~min}$, followed by $95^{\circ} \mathrm{C}$ for $10 \mathrm{sec}, 58^{\circ} \mathrm{C}$ for $30 \mathrm{sec}$ and $72^{\circ} \mathrm{C}$ for $15 \mathrm{sec}$ for a total of 40 cycles. After the reaction was completed, melting curve analysis was performed to identify the specificity of the PCR reaction product. The relative expression of each target gene normalized to GAPDH was analyzed using the $2^{-\Delta \Delta \mathrm{Cq}}$ method (32). The primer sequences are listed in Table II.

Statistical data analysis. All statistical analyses were performed using SPSS v25.0 software (IBM Corp.), and one-way ANOVA followed by Tukey's post hoc test or Kruskal-Wallis followed by Dunn's post hoc test was used. Data are presented as the mean \pm standard deviation or the median (interquartile range) for normally or nonnormally distributed parameters, respectively. $\mathrm{P}<0.05$ was considered to indicate a statistically significant difference.

\section{Results}

Effects of compatibility groups of four effective ingredients on neurological deficits in rats with cerebral IR injury. The neurological deficit of the IRU group was more severe $(\mathrm{P}<0.01)$ than that of the sham group. Compared with the IRU group, the DHI group indicated a significant improvement in the symptoms of neurological deficit $(\mathrm{P}<0.05)$. In addition, all orthogonal compatibility groups were indicated to exhibit an improvement in the symptoms of neurological deficit to different degrees compared with IRU group. Specifically, the symptoms of neurological deficit in the orthogonal groups 4 and 6 were more similar to those in DHI group, and had an improved neurological score compared with the other orthogonal groups. These results are presented in Table III. 
Table II. Primer sequences of selected genes designed for reverse transcription-quantitative PCR.

\begin{tabular}{lll}
\hline Gene & \multicolumn{1}{c}{ Forward primer (5'-3') } & Reverse primer $\left(5^{\prime}-3^{\prime}\right)$ \\
\hline Apaf-1 & TGGATGAAGCCATGTCCATA & TCCCAGAGAACACACAGCAC \\
Cytochrome $c$ & AAGACTGGACCAAACCTCCA & CTCCATCAGGGTATCCTCTCC \\
Caspase-9 & GCCTCATCATCAACAACGTG & CTTCACCTCCACCATGAAGC \\
Caspase-3 & CTGGACTGCGGTATTGAG & GGGTGCGGTAGAGTAAGC \\
p53 & GCTGAGTATCTGGACGACA & CAGGCACAAACACGAACC \\
GAPDH & GGAAATCGTGCGTGACATTA & AGGAAGGAAGGCTGGAAGAG
\end{tabular}

Apaf1, apoptotic peptidase activating factor 1.

Table III. Effects of compatibility groups of four effective ingredients on neurological deficit in rats with cerebral IR injury.

\begin{tabular}{lc}
\hline Group & Neurological score \\
\hline 1 & $2(2-2.25)$ \\
2 & $2(1.75-2)$ \\
3 & $2(2-2)$ \\
4 & $2(1-2)$ \\
5 & $2(1.75-2.25)$ \\
6 & $2(1-2)$ \\
7 & $2(2-2.25)$ \\
8 & $2(2-2.25)$ \\
9 & $2(1.75-2)$ \\
Sham & 0 \\
IRU & $3(2.75-3)^{\mathrm{a}}$ \\
DHI & $1.5(1-2)^{\mathrm{b}}$ \\
\hline
\end{tabular}

Values are expressed as the median (interquartile range) $(n=6)$. ${ }^{\mathrm{a}} \mathrm{P}<0.01$ vs. sham group; ${ }^{\mathrm{b}} \mathrm{P}<0.05$ vs. IRU group. IR, ischemiareperfusion; IRU, IR untreated; DHI, Danhong injection.

Effects of compatibility groups of four effective ingredients on cerebral infarct volume in rats with cerebral IR injury. Following TTC staining, the brain sections of the sham operation group appeared red. The cerebral infarct area of the IRU group was more pronounced $(\mathrm{P}<0.01)$ than that of the sham group. Compared with the IRU group, the infarct volume of the DHI group was observed to be significantly reduced $(\mathrm{P}<0.01)$. In addition, the cerebral infarct volume of each drug group decreased to different degrees. The cerebral infarct volume in the orthogonal compatibility groups 2, 4, 6 and 7 was significantly decreased compared with the sham group $(\mathrm{P}<0.01$ or $\mathrm{P}<0.05)$. These results are presented in Fig. 1.

Effects of compatibility groups of four effective ingredients on pathological alterations of brain tissue in rats with cerebral $I R$ injury. There was no evident pathological damage in the brain tissue of the sham group (Fig. 2A). The structure was normal and clear: The arrangement of cells were tight and uniform, the nucleus was intact and the intercellular space was normal without edema. Typical necrotic foci were observed in the brain tissue of the IRU group (Fig. 2B). Cell edema was visible, the number of cells was reduced, and the arrangement of cells was sparse and disordered. In addition, the boundaries between cells were blurred, the nuclei were atrophied, and a triangular dense nucleus was visible. Compared with the IRU group, brain tissue damage was markedly improved in the DHI group (Fig. 2C). The DHI group presented an increased number of normal neurons and only partial edema degeneration. The orthogonal compatibility of Danshen and Honghua was observed to be most effective in the reduction of pathological tissue damage in groups 2 and 4 . These results are presented in Fig. 2.

Effects of compatibility groups offour effective ingredients on the expression levels of Bcl-2 and Bax in the serum of rats with cerebral IR injury. The serum ratio of Bcl-2/Bax in the IRU group was significantly lower $(\mathrm{P}<0.01)$ than the serum ratio of the sham group. When compared with the IRU group, the DHI group and the orthogonal administration groups (groups 2, 3, 4, 5, 6 and 8) indicated a significant increase in the Bcl-2/Bax ratio $(\mathrm{P}<0.01$ or $\mathrm{P}<0.05)$. In addition, there was no significant difference observed among orthogonal groups (groups 2, 3, 4, $5,6$ and 8 ) and the DHI group ( $\mathrm{P}>0.05)$. However, orthogonal groups 1, 7 and 9 showed statistical difference compared with DHI group $(\mathrm{P}<0.01$ or $\mathrm{P}<0.05)$. The result of group 4 was the closest to that of DHI group, which indicates that group 4 and the DHI group exhibited similar efficacy. These results are presented in Table IV.

Effect of DHI and compatibility groups of four effective ingredients on caspase-3 expression in the CAI area of the hippocampus as detected by immunohistochemistry. Rats in the sham-operated group (Fig. 3A) exhibited low numbers of yellow brown caspase- 3 positive cells in the CA1 area of the hippocampus. When compared with the sham group, the IRU group (Fig. 3B) presented increased cytoplasmic staining of caspase- 3 in the hippocampal $C A 1$ region $(\mathrm{P}<0.01)$. Orthogonal group 4 was observed to exhibit significantly reduced expression of caspase- 3 protein $(\mathrm{P}<0.05)$ when compared with the IRU group. The results are reflected in Fig. 3 and Table V.

Expression levels of cytochrome c, apoptotic peptidase activating factor 1 (apaf-1), caspase-9, caspase-3 and p53 mRNA. RT-qPCR results indicated that the expression level of cytochrome $c$, apaf-1, caspase-9, caspase-3 and p53 mRNA in the 

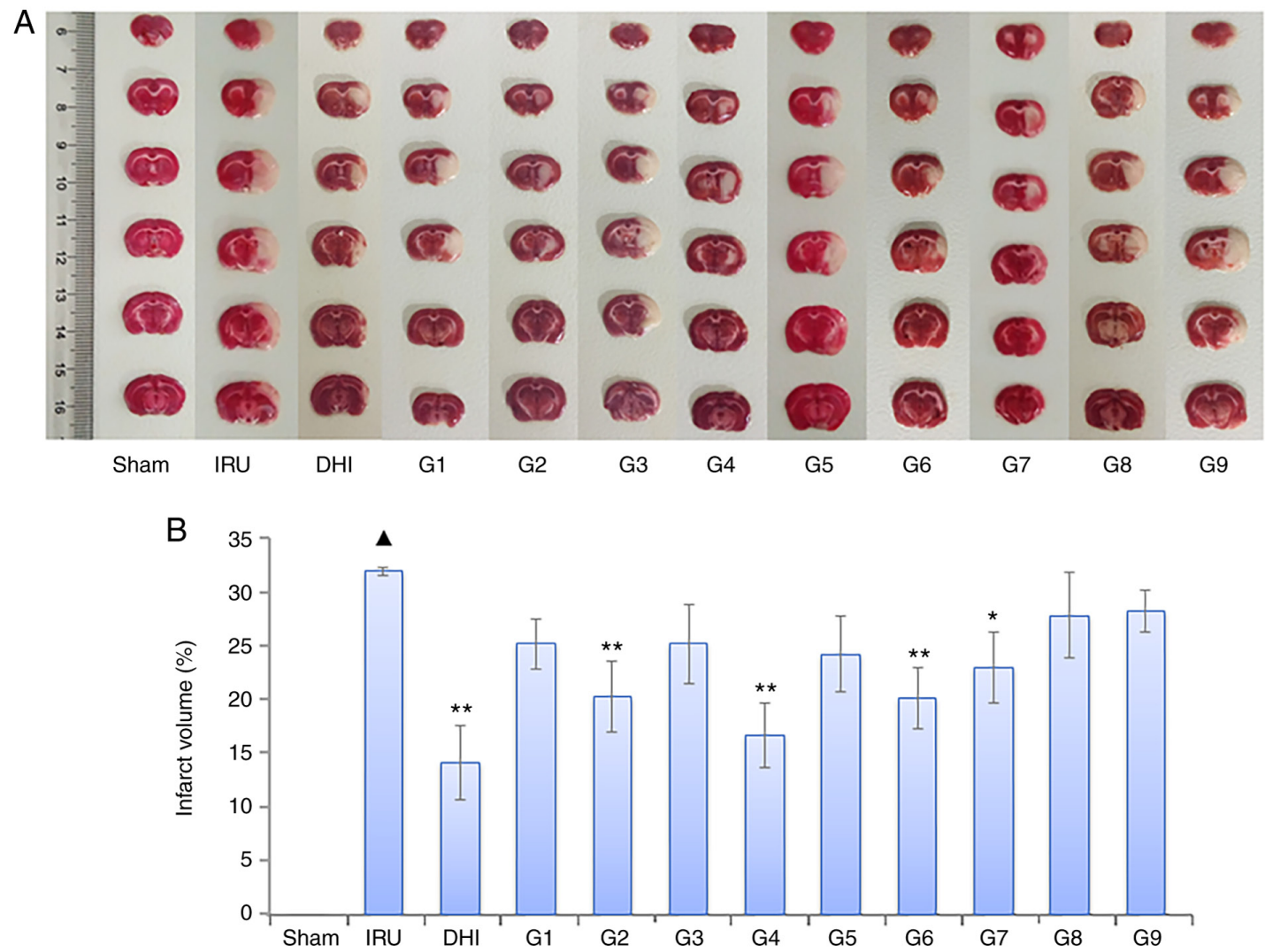

Figure 1. Effect of DHI and orthogonal groups on cerebral infarct volume in rats after focal cerebral IR. Infarct volume was assessed by TTC staining at day 3 after middle cerebral artery occlusion. (A) Representative TTC staining of the cerebral infarct in coronal sections of rat brain. (B) Infarct volumes assessed by TTC staining. The data are presented as the mean \pm standard deviation $(n=6) .{ }^{\wedge} \mathrm{P}<0.01$ vs. sham; ${ }^{*} \mathrm{P}<0.05,{ }^{* *} \mathrm{P}<0.01$ vs. IRU group. IR, ischemia-reperfusion; TTC, 2,3,5-triphenyltetrazolium chloride; IRU, IR untreated; DHI, Danhong injection; G, orthogonal group.

Table IV. Effects of compatibility groups of four effective ingredients on the secretion of $\mathrm{Bcl}-2$ and $\mathrm{Bax}$ in the serum of rats with cerebral IR injury.

\begin{tabular}{lccc}
\hline Group & Bcl-2/ng $\cdot \mathrm{ml}^{-1}$ & Bax $/ \mathrm{ng} \cdot \mathrm{ml}^{-1}$ & Bcl-2/Bax \\
\hline 1 & $97.58 \pm 9.30$ & $6.52 \pm 1.02^{\mathrm{a}}$ & $14.97 \pm 1.43^{\mathrm{b}}$ \\
2 & $109.08 \pm 11.86$ & $5.04 \pm 0.79^{\mathrm{c}}$ & $21.64 \pm 2.35^{\mathrm{c}}$ \\
3 & $102.15 \pm 11.25$ & $5.64 \pm 0.72^{\mathrm{c}}$ & $18.11 \pm 1.99^{\mathrm{d}}$ \\
4 & $117.33 \pm 16.11^{\mathrm{c}}$ & $4.53 \pm 0.52^{\mathrm{c}}$ & $25.90 \pm 3.56^{\mathrm{c}}$ \\
5 & $101.08 \pm 11.88$ & $5.84 \pm 0.93^{\mathrm{c}}$ & $17.31 \pm 2.03^{\mathrm{d}}$ \\
6 & $107.88 \pm 11.06$ & $5.09 \pm 0.60^{\mathrm{c}}$ & $21.19 \pm 2.17^{\mathrm{c}}$ \\
7 & $98.12 \pm 11.32$ & $6.18 \pm 0.84^{\mathrm{b}}$ & $15.88 \pm 1.83^{\mathrm{b}}$ \\
8 & $102.56 \pm 11.70$ & $5.69 \pm 0.66^{\mathrm{c}}$ & $18.02 \pm 2.06^{\mathrm{d}}$ \\
9 & $99.76 \pm 12.82$ & $7.51 \pm 1.05^{\mathrm{a}}$ & $13.28 \pm 1.71^{\mathrm{a}}$ \\
Sham & $149.38 \pm 26.59$ & $3.48 \pm 0.18$ & $42.93 \pm 7.64$ \\
IRU & $82.17 \pm 18.34^{\mathrm{e}}$ & $7.67 \pm 0.75^{\mathrm{e}}$ & $10.71 \pm 2.39^{\mathrm{e}}$ \\
DHI & $121.21 \pm 17.79^{\mathrm{c}}$ & $4.49 \pm 0.39^{\mathrm{c}}$ & $27.00 \pm 3.96^{\mathrm{c}}$ \\
\hline
\end{tabular}

Values are expressed as the mean $\pm \mathrm{SD}(\mathrm{n}=6)$. ${ }^{\mathrm{a}} \mathrm{P}<0.01$ and ${ }^{\mathrm{b}} \mathrm{P}<0.05$ vs. DHI group; ${ }^{\circ} \mathrm{P}<0.01$ and ${ }^{\mathrm{d}} \mathrm{P}<0.05$ vs. IRU group; ${ }^{\mathrm{e}} \mathrm{P}<0.01$ vs. sham group. IR, ischemia-reperfusion; IRU, IR untreated; DHI, Danhong injection.

IRU group was significantly higher than that of the sham group $(\mathrm{P}<0.01)$. Compared with the IRU group, the orthogonal compatibility groups were indicated to exhibit decreased expression
Table V. Effect of compatibility groups of four effective ingredients on caspase- 3 protein expression in rats after cerebral IR injury.

\begin{tabular}{lc}
\hline Group & Caspase-3 \\
\hline 1 & $6(4.75-6.25)$ \\
2 & $4(2.75-5)$ \\
3 & $4(3.75-5)$ \\
4 & $3.5(2.75-4)^{\mathrm{a}}$ \\
5 & $4.5(3.5-6)$ \\
6 & $4.5(3.75-5.25)$ \\
7 & $5(4.75-6)$ \\
8 & $5(3.75-6)$ \\
9 & $5.5(3.75-7)$ \\
Sham & $2(1.75-2)$ \\
IRU & $7(5.5-8)^{\mathrm{b}}$ \\
DHI & $4(2.75-5)$ \\
\hline
\end{tabular}

Values are expressed as the median (interquartile range) $(n=6)$. ${ }^{\mathrm{a}} \mathrm{P}<0.05$ vs. IRU group; ${ }^{\mathrm{b}} \mathrm{P}<0.01$ vs. sham group. IR, ischemia-reperfusion; IRU, IR untreated; DHI, Danhong injection.

level of cytochrome $c$, apaf-1, caspase-9, caspase-3 and p53 mRNA genes. In groups 2, 4 and 6, the expression level of cytochrome $c$, apaf-1, caspase-9, caspase-3 and p53 mRNA was 
A

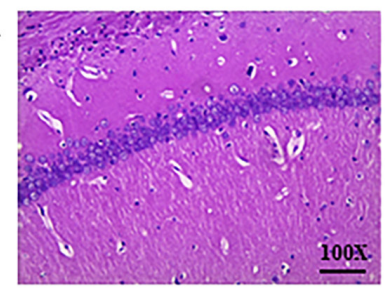

$\mathrm{D}$

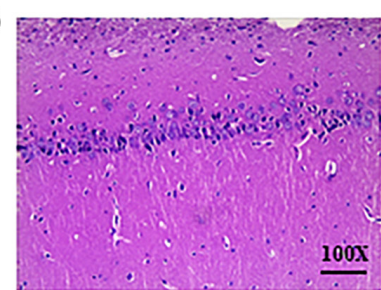

$\mathrm{G}$
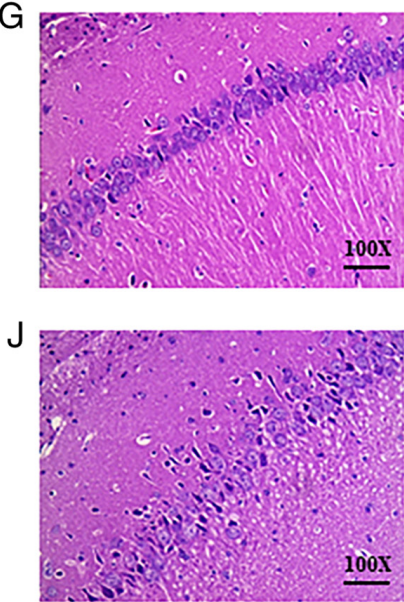

B

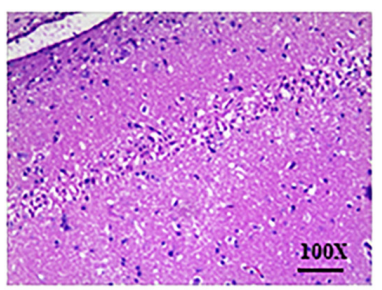

$\mathrm{E}$

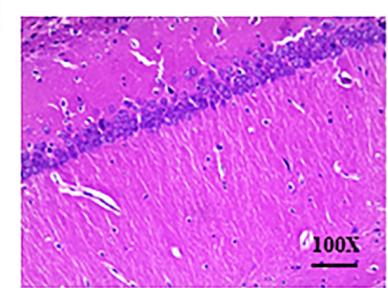

$\mathrm{H}$

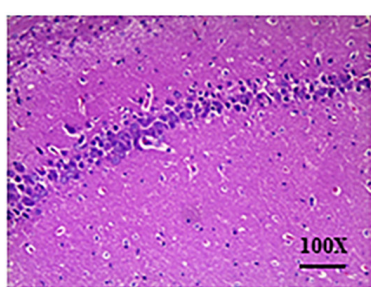

$\mathrm{K}$

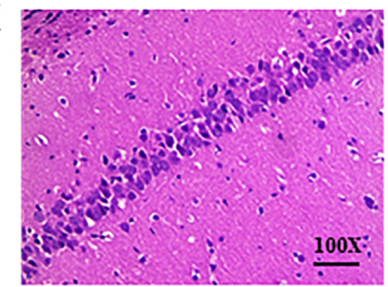

C

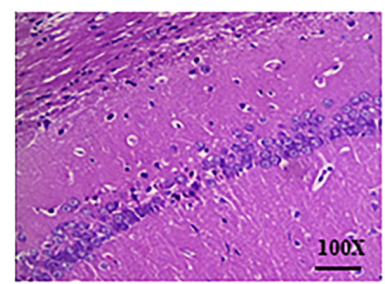

$\mathrm{F}$

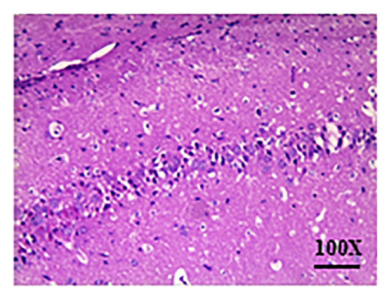

I

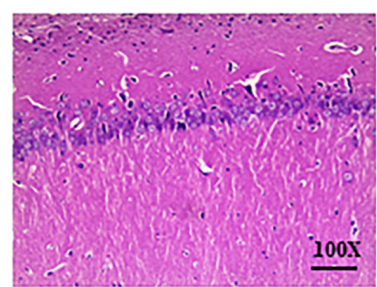

$\mathrm{L}$

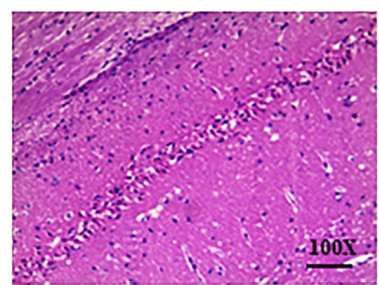

Figure 2. Effect of DHI and compatibility groups of four effective ingredients on brain histopathological alterations in the ischemic penumbra at day 3 of reperfusion after middle cerebral artery occlusion (magnification, x100). (A-L) Sham, ischemia-reperfusion untreated, DHI and orthogonal compatibility $1-9$ groups, respectively $(\mathrm{n}=6)$. DHI, Danhong injection.

significantly decreased $(\mathrm{P}<0.01$ or $\mathrm{P}<0.05)$. When compared with the DHI group, the expression levels of cytochrome $c$ in groups 1, 7 and 8, apaf-1 in groups 7 and 9, caspase-9 in groups 7 and 8, caspase-3 in groups 5, 7 and 9 exhibited significant differences $(\mathrm{P}<0.01$ or $\mathrm{P}<0.05)$. This indicated that the efficacy of the orthogonal compatibility groups 2, 4 and 6 and the DHI group was similar. These results are presented in Table VI.

\section{Discussion}

Known as one of the top four life-threatening diseases (33), strokes are frequent in clinic patients (34). Ischemic strokes are common, accounting for $\sim 87 \%$ of all strokes worldwide $(2,35)$. The incidence of ischemic strokes is higher than that of other types of stroke, which could pose a serious threat to human health (1). Consequently, the prevention and treatment of ischemic stroke and cerebrovascular disease has become a priority throughout the world (36). It is also of great clinical significance and social value to explore the effective treatment methods for patients with ischemic stroke (37).

The compatibility law is one of the core issues in the study of prescription science. It requires a higher level of understanding and generalization of prescription compatibility methods $(36,38)$. This law was a helpful and significant guide for writing clinical prescriptions and further developing the theory of prescription science (37). The study of the compatibility of prescription drugs has been considered important by ancient and modern doctors (38).

Drug pairs are a commonly used compatibility form of TCM clinical prescriptions (38). Drug pairs follow the theory of TCM, including four odors and five flavors, ascents and descents, channel tropism, toxicity and side effects and the principle of complementary or opposite combination (39). A drug pair has the characteristics of a simple structure and clear compatibility effect (8). This theory is the culmination of accumulated clinical medication experience by physicians of past dynasties (40). The present study on the main effective ingredients of Danshen and Honghua as effective prescriptions will help clarify the mechanism of action of these drugs and reveal their useful characteristics (41).

The pathophysiological process of cerebral IR injury is a complex cascade reaction $(2,42)$. The pathogenesis involves a variety of dysregulations, including excitatory amino acid toxicity, intracellular calcium overload, excessive formation of oxygen free radicals, cascade free radical chain reactions, inflammatory reactions, mitochondrial dysfunction and apoptosis (42-45). These events can ultimately cause irreversible brain injury (46). A notable cause of IR injury is the increased apoptosis of local neurons after the initial cerebral ischemia (44). 
Table VI. Effect of compatibility groups of four effective ingredients on the expression levels of cytochrome $c$, apaf-1, caspase-9, caspase- 3 and p53 in rats after cerebral IR injury.

\begin{tabular}{|c|c|c|c|c|c|}
\hline Group & Cytochrome $c$ & Apaf-1 & Caspase-9 & Caspase-3 & p53 \\
\hline 1 & $3.64 \pm 0.86^{\mathrm{a}}$ & $2.69 \pm 0.60$ & $2.43 \pm 0.67$ & $2.69 \pm 0.68$ & $2.61 \pm 0.54$ \\
\hline 2 & $2.32 \pm 0.57^{b}$ & $2.25 \pm 0.51^{\mathrm{c}}$ & $1.98 \pm 0.54^{\mathrm{b}}$ & $1.73 \pm 0.36^{\mathrm{b}}$ & $2.14 \pm 0.40^{\mathrm{b}}$ \\
\hline 3 & $3.18 \pm 0.61$ & $2.76 \pm 0.72$ & $2.64 \pm 0.76$ & $2.51 \pm 0.57$ & $2.55 \pm 1.05$ \\
\hline 4 & $2.02 \pm 0.56^{b}$ & $1.86 \pm 0.57^{\mathrm{b}}$ & $2.01 \pm 0.48^{\mathrm{b}}$ & $1.90 \pm 0.53^{b}$ & $2.32 \pm 0.71^{\mathrm{c}}$ \\
\hline 5 & $2.31 \pm 0.59^{b}$ & $2.65 \pm 0.65$ & $2.61 \pm 0.72$ & $2.84 \pm 0.39^{\mathrm{d}}$ & $2.85 \pm 1.05$ \\
\hline 6 & $2.46 \pm 0.63^{b}$ & $1.95 \pm 0.49^{b}$ & $1.91 \pm 0.56^{\mathrm{b}}$ & $1.92 \pm 0.70^{\mathrm{b}}$ & $2.41 \pm 1.14^{\mathrm{c}}$ \\
\hline 7 & $3.31 \pm 0.74^{\mathrm{d}}$ & $3.08 \pm 0.66^{\mathrm{d}}$ & $2.88 \pm 0.40^{\mathrm{d}}$ & $2.84 \pm 0.44^{\mathrm{d}}$ & $2.26 \pm 1.26^{c}$ \\
\hline 8 & $3.70 \pm 0.67^{\mathrm{a}}$ & $2.94 \pm 0.91$ & $2.96 \pm 0.66^{\mathrm{d}}$ & $2.78 \pm 0.79$ & $2.65 \pm 1.02$ \\
\hline 9 & $2.74 \pm 0.61^{b}$ & $3.09 \pm 0.78^{\mathrm{d}}$ & $2.69 \pm 0.73$ & $2.96 \pm 0.70^{\mathrm{d}}$ & $2.96 \pm 0.99$ \\
\hline Sham & $1.01 \pm 0.18$ & $1.02 \pm 0.21$ & $1.01 \pm 0.18$ & $1.01 \pm 0.18$ & $1.02 \pm 0.26$ \\
\hline IRU & $4.45 \pm 0.86^{\mathrm{e}}$ & $3.65 \pm 0.79^{\mathrm{e}}$ & $3.43 \pm 0.85^{\mathrm{e}}$ & $3.43 \pm 0.85^{\mathrm{e}}$ & $4.22 \pm 0.78^{\mathrm{e}}$ \\
\hline DHI & $1.75 \pm 0.51^{\mathrm{b}}$ & $1.75 \pm 0.51^{b}$ & $1.69 \pm 0.27^{b}$ & $1.69 \pm 0.27^{\mathrm{b}}$ & $2.11 \pm 0.73^{b}$ \\
\hline
\end{tabular}

Values are expressed as the mean $\pm \mathrm{SD}(\mathrm{n}=6) .{ }^{\mathrm{a}} \mathrm{P}<0.01$ and ${ }^{\mathrm{d}} \mathrm{P}<0.05$ vs. $\mathrm{DHI}$ group; ${ }^{\mathrm{b}} \mathrm{P}<0.01$ and ${ }^{\mathrm{C}} \mathrm{P}<0.05$ vs. IRU group; ${ }^{\mathrm{e}} \mathrm{P}<0.01 \mathrm{vs}$. sham group. IR, ischemia-reperfusion; IRU, IR untreated; DHI, Danhong injection; Apaf-1, apoptotic peptidase activating factor 1.

\section{A}

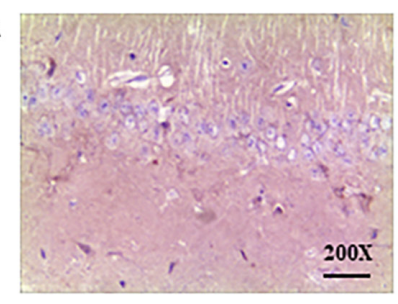

D

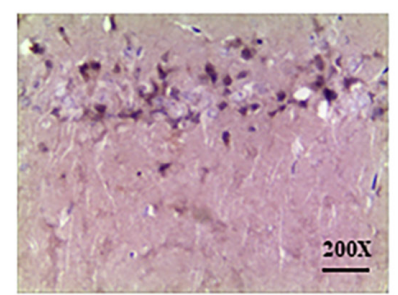

$G$

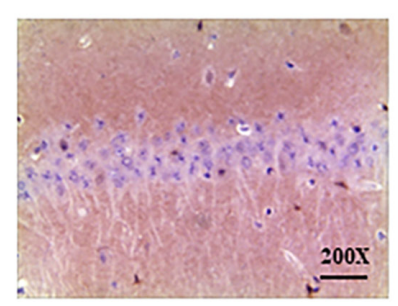

$\mathrm{J}$

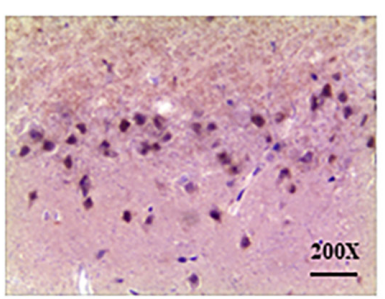

B

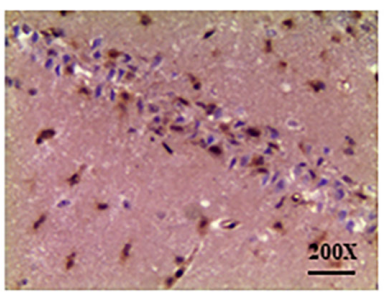

E

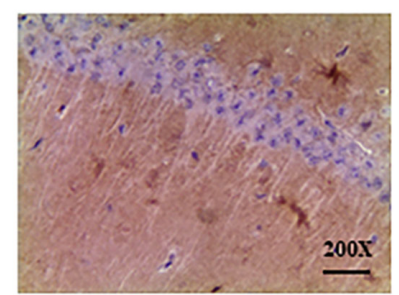

$\mathrm{H}$

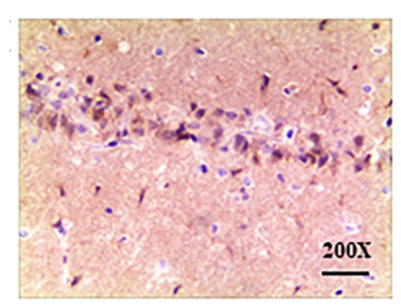

$\mathrm{K}$

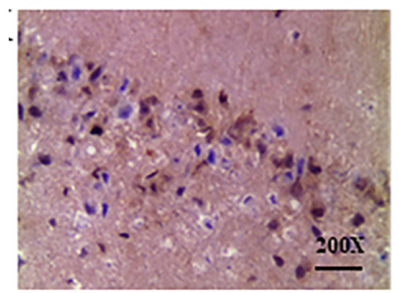

C

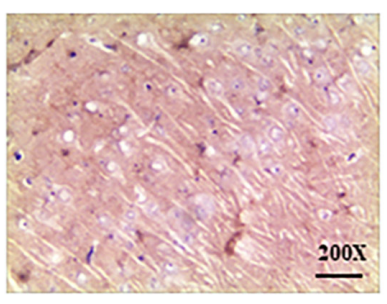

$\mathrm{F}$

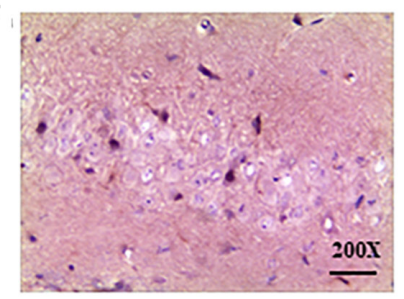

।

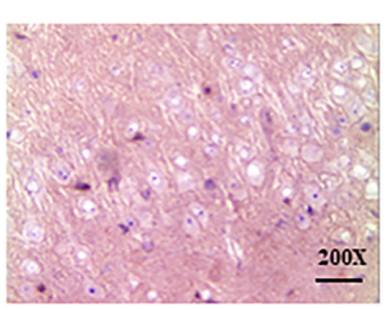

$\mathrm{L}$

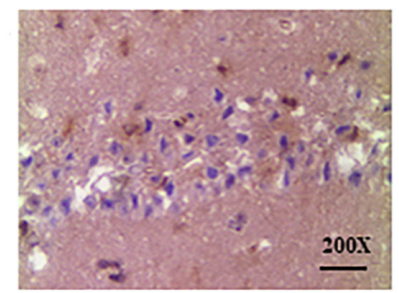

Figure 3. Effect of DHI and compatibility groups of four effective ingredients on caspase-3 protein expression in rats after cerebral IR injury (magnification, x200). (A-L) Sham, IR untreated, DHI and orthogonal compatibility 1-9 groups, respectively (n=6). IR, ischemia-reperfusion; DHI, Danhong injection.

The regulation of factors and signal transduction pathways involved in neuronal apoptosis reduces the degree of brain injury during ischemia and prevents further development of apoptosis $(44,45)$. This encourages the possibility of a breakthrough 
in the treatment of cerebrovascular diseases. The caspase family serves an important role in the apoptotic process of neurons $(47,48)$. This family of proteins represents the common pathway for the final implementation of apoptosis (49).

Cerebral ischemia and hypoxia can initiate a series of pathological changes within cells (42). One important response is the opening of a permeability transition pore that activates the endogenous apoptotic pathway (50). The precursors of caspase-9, procaspase- 9 and cytochrome $c$ are then released from the mitochondria to form apoptotic bodies with apaf-1 (47,51). These apoptotic bodies activate caspase-9 and downstream caspase-3, which causes apoptosis $(50,51)$.

The damage of the mitochondrial membrane is also closely associated with Bcl-2 family members, including Bcl-2, Bax and $\mathrm{Bad}$ (52). These proteins are involved in the regulation of apoptosis (53). Bcl-2 and Bax are a group of channel proteins, which can affect the state of cells by regulating the permeability of the mitochondrial membrane. Specifically, Bax can regulate the permeability of the mitochondrial extracorporeal membrane, causing increased release of cytochrome $c$ from the mitochondria and promotion of apoptosis $(54,55)$. Bcl-2 inhibits the activation of the caspase family and halts apoptosis by preventing the formation of the Bax channel (56-58). The p53 protein is a key molecule in promoting neuronal apoptosis (59), which can upregulate Bax and downregulate Bcl-2 (51,54). It can also cause a caspase family cascade reaction and promote cell apoptosis $(47,60)$.

The present study has several limitations. Firstly, the experimental period in the present research was 3 days as a result of the small treatment time. Therefore, the efficacy of drug treatment for 1,5 and 7 days was not examined. Secondly, as oxidative stress and mitochondrial dysfunction are upstream factors leading to apoptosis, a further study could evaluate the comprehensive and in-depth effect of these pathways regulated by the combination of Danshen and Honghua after cerebral IR injury.

The present study indicated that the expression levels of apoptosis-related factors, such as cytochrome $c$, apaf-1, caspase-9, caspase-3 and p53, were significantly increased after cerebral IR injury. In addition, the damage of hippocampal cells was improved to varying degrees after drug treatment. These findings suggested that the combination of Danshen and Honghua exhibited a protective effect on rats after cerebral IR injury. In addition, orthogonal group $4(30 \mathrm{mg} / \mathrm{kg}$ tanshinol; $2.5 \mathrm{mg} / \mathrm{kg}$ salvianolic acid $\mathrm{A} ; 16 \mathrm{mg} / \mathrm{kg}$ salvianolic acid B; and $8 \mathrm{mg} / \mathrm{kg}$ hydroxysafflor yellow $\mathrm{A}$ ) exhibited a significant inhibition of apoptosis. These drugs may function by inhibiting key targets upstream of caspase- 3 to prevent apoptosis. Ultimately, the effective and compatible ingredients of Danshen and Honghua were revealed to exhibit a significant protective effect on cerebral IR injury in rats.

\section{Acknowledgements}

Not applicable.

\section{Funding}

This work was supported by National Key R\&D Projects of China (grant. nos. 2019YFC1708600 and 2019YFC1708604),
Zhejiang Provincial Natural Science Foundation of China (grant. no. LQ19H270001), National Natural Science Foundation of China (grant. no. 81874366), Key Laboratory of TCM Encephalopathy of Zhejiang Province (grant. no. 2020E10012) and Open Foundation of Scientific Research of Zhejiang Chinese Medical University (no. ZYX2018009).

\section{Availability of data and materials}

The datasets used and/or analyzed during the current study are available from the corresponding author on reasonable request.

\section{Authors' contributions}

JY and HZ conceived the idea and designed the study. HW and LC performed the experiments. ZD and ZL established the cerebral IR model in rats. ZL wrote the manuscript. YY and HW participated in the data acquisition and statistical analysis, and YY revised the manuscript. All authors have read and approved the final manuscript. JY and HZ confirm the authenticity of all the raw data.

\section{Ethics approval and consent to participate}

Animal welfare and experiments were strictly in accordance with the Regulation for the Administration of Affairs Concerning Experimental Animals (State Science and Technology Commission, 1988) and approved by the Institutional Animal Care and Use Committee of Zhejiang Laboratory Animal Center (Hangzhou, China).

\section{Patient consent for publication}

Not applicable.

\section{Competing interests}

The authors declare that they have no competing interests.

\section{References}

1. Gao L, Song Z, Mi J, Hou P, Xie C, Shi J, Li Y and Manaenko A: The effects and underlying mechanisms of cell therapy on blood-brain barrier integrity after ischemic stroke. Curr Neuropharmacol 18: 1213-1226, 2020.

2. Ajoolabady A, Wang S, Kroemer G, Penninger JM, Uversky VN Pratico D, Henninger N, Reiter RJ, Bruno A, Joshipura K, et al: Targeting autophagy in ischemic stroke: From molecular mechanisms to clinical therapeutics. Pharmacol Ther 3: 107848, 2021.

3. Shekhar S, Liu Y, Wang S, Zhang H, Fang X, Zhang J, Fan L, Zheng B, Roman RJ, Wang Z, et al: Novel mechanistic insights and potential therapeutic impact of trpc6 in neurovascular coupling and ischemic stroke. Int J Mol Sci 22: 2074, 2021.

4. Radu RA, Terecoasă EO, Băjenaru OA and Tiu C: Etiologic classification of ischemic stroke: Where do we stand? Clin Neurol Neurosurg 159: 93-106, 2017.

5. Writing Group Members; Mozaffarian D, Benjamin EJ, Go AS, Arnett DK, Blaha MJ, Cushman M, Das SR, de Ferranti S, Després JP, et al: Heart disease and stroke statistics-2016 update: A report from the American heart association. Circulation 26: e38-e360, 2016.

6. Orellana-Urzúa S, Rojas I, Líbano L and Rodrigo R: Pathophysiology of ischemic stroke: Role of oxidative stress. Curr Pharm Des 26: 4246-4260, 2020. 
7. Wang Z, Wan H, Tong X, He Y, Yang J, Zhang L, Shao C, Ding Z, Wan $\mathrm{H}$ and Li C: An integrative strategy for discovery of functional compound combination from traditional Chinese medicine: Danhong injection as a model. Biomed Pharmacother 138: 111451,2021

8. Cui Y, Liu X, Li X and Yang H: In-Depth proteomic analysis of the hippocampus in a rat model after cerebral ischaemic injury and repair by danhong injection (DHI). Int J Mol Sci 18: 1335, 2017.

9. Gu S, Ma Y, Ge K, Nie R, Wu E and Li Y: Danshen-Honghua ameliorates stress-induced menopausal depression in rats. Neural Plast 2018: 1-5, 2018.

10. Cheng Q, Pu ZJ, Zhou GS, Wang J, Zhu ZH, Yue SJ, Li JP, Shang LL, Tang YP, Shi XQ, et al: Comparative analysis of main bio-active components in the herb pair danshen-honghua and its single herbs by ultra-high performance liquid chromatography coupled to triple quadrupole tandem mass spectrometry. J Sep Sci 40: 3392-3401, 2017.

11. Wang YL, Zhang Q, Yin SJ, Cai L, Yang YX, Liu WJ, Hu YJ, Chen $\mathrm{H}$ and Yang FQ: Screening of blood-activating active components from Danshen-Honghua herbal pair by spectrumeffect relationship analysis. Phytomedicine 54: 149-158, 2019.

12. Meng X, Jiang J, Pan H, Wu S, Wang S, Lou Y and Fan G: Preclinical absorption, distribution, metabolism, and excretion of sodium danshensu, one of the main water-soluble ingredients in salvia miltiorrhiza, in rats. Front Pharmacol 10: 554, 2019.

13. Chien MY, Chuang CH, Chern CM, Liou KT, Liu DZ, Hou YC and Shen YC: Salvianolic acid A alleviates ischemic brain injury through the inhibition of inflammation and apoptosis and the promotion of neurogenesis in mice. Free Radic Biol Med 99: 508-519, 2016

14. Zhao R, Liu X, Zhang L, Yang H and Zhang Q: Current progress of research on neurodegenerative diseases of salvianolic acid B. Oxid Med Cell Longev 24: 3281260, 2019.

15. Lu Y, Yanhong D, Zheng Z, He W, Xia M, Zhang Q and Cao X Hydroxysafflor yellow A (HSYA) improves learning and memory in cerebral ischemia reperfusion-injured rats via recovering synaptic plasticity in the hippocampus. Front Cell Neurosci 12: 371,2018

16. Sun Y, Xu DP, Qin Z, Wang PY, Hu BH, Yu JG, Zhao Y, Cai B, Chen YL, Lu M, et al: Protective cerebrovascular effects of hydroxysafflor yellow A (HSYA) on ischemic stroke. Eur J Pharmacol 818: 604-609, 2018.

17. Xu H, Liu T, Wang W, Su N, Yang L, Yang Z, Dou F, Cui J, Fei F, Ma J, et al: Proteomic analysis of hydroxysafflor yellow A against cerebral ischemia/reperfusion injury in rats. Rejuvenation Res 22: 503-512, 2019.

18. Yang Y, Wang L, Wu Y, Su D, Wang N, Wang J, Shi C, Lv L and Zhang S: Tanshinol suppresses inflammatory factors in a rat model of vascular dementia and protects LPS-treated neurons via the MST1-FOXO3 signaling pathway. Brain Res 1646: 304-314, 2016.

19. Wei ZZ, Chen D, Liu LP, Gu X, Zhong W, Zhang YB, Wang Y, $\mathrm{Yu}$ SP and Wei L: Enhanced neurogenesis and collaterogenesis by sodium danshensu treatment after focal cerebral ischemia in mice. Cell Transplant 4: 622-636, 2018

20. Zhang W, Song JK, Zhang X, Zhou QM, He GR, Xu XN, Rong Y, Zhou WX and Du GH: Salvianolic acid A attenuates ischemia reperfusion induced rat brain damage by protecting the blood brain barrier through MMP-9 inhibition and anti-inflammation. Chin J Nat Med 16: 184-193, 2018.

21. Feng SQ, Aa N, Geng JL, Huang JQ, Sun RB, Ge C, Yang ZJ, Wang LS, Aa JY and Wang FJ: Pharmacokinetic and metabolomic analyses of the neuroprotective effects of salvianolic acid A in a rat ischemic stroke model. Acta Pharmacol Sinica 11: 1435-1444, 2017.

22. Ling C, Liang J, Zhang C, Li R, Mou Q, Qin J, Li X and Wang J: Synergistic effects of salvianolic acid B and puerarin on cerebral ischemia reperfusion injury. Molecules 3: 564, 2018.

23. Yu L, Wan H, Jin W, Yang J, Li C, Dai L, Ge L, Zhou H, Wan H and He Y: Protective effects of effective ingredients of danshen (Radix Salviae Miltiorrhizae) and honghua (Flos Carthami) compatibility after rat hippocampal neurons induced by hypoxia injury. J Tradit Chin Med 38: 685-697, 2018.

24. Longa EZ, Weinstein PR, Carlson S and Cummins R: Reversible middle cerebral artery occlusion without craniectomy in rats. Stroke 20: 84-91, 1989

25. Lu Z, Cao H, Liu D, Zheng Y, Tian C, Liu S, Quan J, Shi L, Liu J and Yu L: Optimal combination of anti-inflammatory components from Chinese medicinal formula Liang-Ge-San. J Ethnopharmacol 269: 113747, 2021.
26. Shao J, Liu Z, Wang L, Song Z, Chang H, Han N and Yin J: Screening of the optimized prescription from suqingwan in terms of its therapeutic effect on DSS-induced ulcerative colitis by its regulation of inflammatory and oxidative mediators. J Ethnopharmacol 18: 54-62, 2017.

27. Alawieh A, Zhao J and Feng W: Factors affecting post-stroke motor recovery: Implications on neurotherapy after brain injury. Behav Brain Res 340: 94-101, 2018.

28. Yasar U: Two-Sided action of danshen on cytoprotective endogenous substances, epoxyeicosatrienoic acids. Chem Biol Interact 291: 152, 2018

29. Zou JB, Zhang XF, Wang J, Wang F, Cheng JX, Yang FY, Song X, Wang Y, Liang YL and Shi YJ: The therapeutic efficacy of danhong injection combined with percutaneous coronary intervention in acute coronary syndrome: A systematic review and meta-analysis. Front Pharmacol 9: 550, 2018.

30. Simani L, Naderi N, Khodagholi F, Mehrpour M and Nasoohi S: Association of long-term atorvastatin with escalated stroke-induced neuroinflammation in rats. J Mol Neurosci 61: 32-41, 2017.

31. Beilner D, Kuhn C, Kost BP, Vilsmaier T, Vattai A, Kaltofen T, Mahner S, Schmoeckel E, Dannecker C, Jückstock J,et al: Nuclear receptor corepressor (NCoR) is a positive prognosticator for cervical cancer. Arch Gynecol Obstet 16: 1007, 2021.

32. Livak KJ and Schmittgen TD: Analysis of relative gene expression data using real-time quantitative PCR and the 2(-Delta DeltaC(T)) method. Methods 25: 402-408, 2001.

33. Jin Y, Pu ZJ, Tang YP, Shang EX, Shi XQ, Juan S, Pang H and Duan J: Effect of promoting blood circulation of herb pair containing Angelicae Sinensis Radix and CarthamSi Flos. Chinese Traditional and Herbal Drugs 48: 2087-2092, 2017.

34. Vavers E, Zvejniece L, Svalbe B, Volska K, Makarova E, Liepinsh E, Rizhanova K, Liepins V and Dambrova M: The neuroprotective effects of R-phenibut after focal cerebral ischemia. Pharmacol Res 113: 796-801, 2016.

35. Katan M and Luft A: Global burden of stroke. Semin Neurol 38 208-211, 2018.

36. Culman J, Nguyen-Ngoc M, Glatz T, Gohlke P, Herdegen T and Zhao Y: Treatment of rats with pioglitazone in the reperfusion phase of focal cerebral ischemia: A preclinical stroke trial. Exp Neurol 238: 243-253, 2012.

37. Decano JL, Viereck JC, Mckee AC, Hamilton JA, Ruiz-Opazo N and Herrera VLM: Early-Life sodium exposure unmasks susceptibility to stroke in hyperlipidemic, hypertensive heterozygous Tg25 rats transgenic for human cholesteryl ester transfer protein. Circulation 119: 1501-1509, 2009.

38. Wang Y, Yang H, Chen L, Jafari M and Tang J: Network-Based modeling of herb combinations in traditional Chinese medicine. Brief Bioinform 8: 1093, 2021.

39. Zhong LY, Cui MN, Yang M and Gong QF: Modern researches on effect of processing of Chinese herb medicine on Chinese medical properties. Zhongguo Zhong Yao Za Zhi 44: 5109-5113, 2019 (In Chinese)

40. Guo Zl, Zhu Y, Su Xt, Liu J, Yang Qx, Nan Jy, Zhao Bc, Zhang Yy, $\mathrm{Yu} \mathrm{Yn}, \mathrm{Li} \mathrm{B}$, et al: DanHong injection dose-dependently varies amino acid metabolites and metabolic pathways in the treatment of rats with cerebral ischemia. Acta Pharmacol Sin 36: 748-757, 2015.

41. Fei YX, Wang SQ, Yang LJ, Qiu YY, Li YZ, Liu WY, Xi T, Fang WR and Li YM: Salvia miltiorrhiza bunge (Danshen) extract attenuates permanent cerebral ischemia through inhibiting platelet activation in rats. J Ethnopharmacol 207: 57-66, 2017.

42. Yang JL, Mukda S and Chen SD: Diverse roles of mitochondria in ischemic stroke. Redox Biol 16: 263-275, 2018.

43. Ouyang YB and Giffard RG: Cellular neuroprotective mechanisms in cerebral ischemia: $\mathrm{Bcl}-2$ family proteins and protection of mitochondrial function. Cell Calcium 36: 303-311, 2004

44. SethL: Apoptosis and brain ischaemia.Prog Neuropsychopharmacol Biol Psychiatry 27: 267-282, 2003.

45. Gupta S, Sharma U, Jagannathan NR and Gupta YK: Neuroprotective effect of lercanidipine in middle cerebral artery occlusion model of stroke in rats. Exp Neurol 288: 25-37, 2017.

46. Wang Y, Zhan G, Cai Z, Jiao B, Zhao Y, Li S and Luo A: Vagus nerve stimulation in brain diseases: Therapeutic applications and biological mechanisms. Neurosci Biobehav Rev 21: 37-53, 2021.

47. Mouw G, Zechel JL, Zhou Y, Lust WD, Selman WR and Ratcheson RA: Caspase-9 inhibition after focal cerebral ischemia improves outcome following reversible focal ischemia. Metab Brain Dis 17: 143-151, 2002. 
48. Marcel V, Fernandes K, Terrier O, Lane DP and Bourdon JC: Modulation of $\mathrm{p} 53 \beta$ and $\mathrm{p} 53 \gamma$ expression by regulating the alternative splicing of TP53 gene modifies cellular response. Cell Death Differ 21: 1377-1387, 2014.

49. Li L, Su Z, Zou Z, Tan H, Cai D, Su L and Gu Z: Ser46 phosphorylation of p53 is an essential event in prolyl-isomerase pin1-mediated p53-independent apoptosis in response to heat stress. Cell Death Dis 4: 96, 2019.

50. Bai X, Tan TY, Li YX, Li Y Chen YF, Ma R, Wang SY, Li Q and Liu ZQ: The protective effect of cordyceps sinensis extract on cerebral ischemic injury via modulating the mitochondrial respiratory chain and inhibiting the mitochondrial apoptotic pathway. Biomed Pharmacother 124: 109834, 2020.

51. Hong B, Van Den Heuvel AP, Prabhu VV, Zhang S and El-Deiry WS: Targeting tumor suppressor $\mathrm{p} 53$ for cancer therapy: Strategies, challenges and opportunities. Curr Drug Targets 15: 80-89, 2014.

52. Birkinshaw RW and Czabotar PE: The BCL-2 family of proteins and mitochondrial outer membrane permeabilisation. Semin Cell Dev Biol 72: 152-162, 2017.

53. Cheng CY, Kao ST and Lee YC: Ferulic acid exerts anti-apoptotic effects against ischemic injury by activating HSP70/Bcl-2- and HSP70/autophagy-mediated signaling after permanent focal cerebral ischemia in rats. Am J Chin Med 47: 39-61, 2019.

54. Enomoto A, Yamada J, Morita A and Miyagawa K: Bisdemethoxycurcumin enhances X-ray-induced apoptosis possibly through $\mathrm{p} 53 / \mathrm{Bcl}-2$ pathway. Mutat Res 815: 1-5, 2017.
55. Jiang J, Dai J and Cui H: Vitexin reverses the autophagy dysfunction to attenuate MCAO-induced cerebral ischemic stroke via mTOR/Ulk1 pathway. Biomed Pharmacother 99: 583-590, 2018.

56. Czabotar PE, Lessene G, Strasser A and Adams JM: Control of apoptosis by the BCL-2 protein family: Implications for physiology and therapy. Nat Rev Mol Cell Biol 15: 49-63, 2014.

57. Anilkumar U and Prehn JH: Anti-Apoptotic BCL-2 family proteins in acute neural injury. Front Cell Neurosci 8: 281, 2014.

58. D'Orsi B, Matekya J and Prehn JHM: Control of mitochondrial physiology and cell death by the Bcl-2 family proteins bax and bok. Neurochem Int 109: 162-170, 2017.

59. Lim Y, Dorstyn L and Kumar S: The p53-caspase-2 axis in the cell cycle and DNA damage response. Exp Mol Med 53: 517-527, 2021.

60. Zhang L, Zhao H, Zhang X, Chen L, Zhao X, Bai X and Zhang J: Nobiletin protects against cerebral ischemia via activating the p-Akt, p-CREB, BDNF and Bcl-2 pathway and ameliorating BBB permeability in rat. Brain Res Bull 96: 45-53, 2013.

This work is licensed under a Creative Commons Attribution-NonCommercial-NoDerivatives 4.0 International (CC BY-NC-ND 4.0) License. 